\title{
Marie Dentière et la prédication des femmes
}

CYNTHIA

SKENAZI

Résumé: Dans son Epistre très utile (1539), Marie Dentière s'approprie le modèle de la prédication donné par Farel pour dénoncer avec fougue la corruption de l'Église romaine. La réaction de Farel et de Calvin à l'activité d'une femme qui, en outre, contrairement aux interdits religieux, prêche en public à Genève est moins négative qu'il n'y paraît. Dans ce sens, l'oeuvre de cette ancienne abbesse de Tournai, excommuniée, pourrait avoir constitué un premier pas vers la remise en cause du silence des femmes dans l'Église.

Qi l'activité des femmes dans la diffusion de la Réforme a fait l'objet de $\checkmark$ nombreux travaux, le cas de Marie Dentière n'a pas suffisamment retenu l'attention des exégètes. ${ }^{1}$ Il constitue cependant, à bien des égards, une exception particulièrement intéressante. Par contraste avec les rares prédicantes françaises de la première moitiè du seizième siècle, cette abbesse de Tournai quitte son couvent pour défendre publiquement sa foi à Genève. Le ton de son Epistre très utile (1539) distingue en outre cette oeuvre de la pratique courante de l'édification: le texte de Dentière dénonce avec une violence extrême l'hypocrisie ambiante et la corruption du clergé genevois. Cette agressivité a déjà été soulignée par les commentateurs, mais ses présupposés sont largement passés inaperçus. Dans l'Epistre très utile, Marie Dentière ne se limite pas à justifier par l'exemple le droit des femmes à prêcher en public, mais s'approprie, comme je voudrais le suggérer dans cet article, le modèle de la prédication donné par Guillaume Farel. Je me propose de dégager les implications de cette démarche et de suivre les réactions qu'elle a suscitées dans l'entourage de Dentière. Il s'agira notamment de s'interroger sur l'attitude de Farel et de 
Calvin devant cette prise de la parole puisque, conformément aux règles imposées par l'Église, tous deux interdisent formellement aux femmes l'exercice de la prédication.

\section{La prise de la parole}

Rappelons brièvement quelques faits qui permettront de situer l'Epistre très utile. Première femme de France à être excommuniée, Marie Dentière quitte son couvent des Augustins de Tournai vers le milieu des années 1520, épouse un prêtre, Simon Robert, et s'établit avec lui à Strasbourg. En 1526, le couple fait la connaissance de Guillaume Farel et suit le prédicateur à Aigle avant de revenir à Strasbourg. Deux ans plus tard, Robert devient pasteur a Bex. À sa mort, sa femme épouse Antoine Froment, un proche auxiliaire de Farel. En mars 1535, Marie Dentière et ses cinq enfants rejoignent Farel et Froment à Genève. L'ancienne abbesse prend part à la propagande religieuse en exhortant les Clarisses à se convertir à la foi nouvelle et à se marier.

Attribuée à Dentière par Albert Rilliet à la fin du siècle dernier, La guerre et deslivrance de la ville de Genesve fidèlement faicte et composée par ung Marchant demourant en icelle (1536) relate les événements qui aboutissent en 1536 au triomphe de la Réforme dans la cité. ${ }^{2}$ Le ton belliqueux de ce récit des troubles populaires, des discussions théologiques, de l'activité des prédicateurs constitue, selon Rilliet, une des raisons majeures de faire de Dentière l'auteur de cette oeuvre anonyme. Personne n'a depuis lors contesté cette hypothèse qui s'appuie aussi sur des rapprochements entre certains passages de La guerre et deslivrance et un texte de l'ancienne abbesse publié trois ans plus tard. Vers la fin avril 1539, le même imprimeur Jean Gérard fait en effet paraître à Genève l'Epistre très vtile faicte \& composée par vne femme Chrestienne de Tournay, Envoyée à la Royne de Nauarre seurdu Royde France. Contre Lés Turcz, Iuifz, Infideles, Faulxchrestiens, Anabaptistes \& Lutheriens. D’après les manuscrits de Froment, l'ouvrage répondait aux questions de Marguerite de Navarre sur le sort d' "une sienne commère nommée Marie Dentière" et sur les raisons pour lesquelles "les ministres de la parolle de Dieu avaient été deschassés."3 L'Epistre très utile confirme cette indication: d'entrée de jeu, l'auteurs'adresse à la reine de Navarre et lui demande d'intercéder auprès de François $1^{\text {er }}$ pour mettre fin aux divisions qui déchirent la France.

Signé des initiales “M. D.,” ce texte suscite aussitôt un succès de scandale. Sa sympathie manifeste pour les idées religieuses de Guillaume Farel et de Jean Calvin (exilés de Genève depuis avril 1538) ainsi que la critique 
véhémente de certains prédicants de la ville n'échappent pas à la vigilance de ces derniers: ils portent aussitôt plainte au Conseil. Malgré la fausse indication “à Anuers chez Martin l'empereur," Gérard est arrêté et jugé; le livre est retiré de la circulation. Cette mesure semble avoir été mal appliquée puisqu'un an plus tard une lettre de Froment mentionne l'existence de plusieurs centaines d'exemplaires. Tout au long du procès, le tribunal attribue l'oeuvre à Froment, sans citer une seule fois le nom de son épouse, alors que Froment lui-même fait de celle-ci l'auteur de l'Epistre. ${ }^{4}$ Le rôle de Dentière dans la composition est d'ailleurs attesté par des témoignages de l'époque: la Bibliotheque Françoise de La Croix du Maine la désigne comme l'auteur de cet ouvrage; un texte de Valère André fait en outre allusion à Maria d'Entières, Tornacensis, mulier docta, claruit anno 1539, quo vulgavit, Gallice scriptam, Epistolam contre Turcas, Iudaeos, Infideles, Pseudo-Christianos, anabaptistas et Lutheranos. ${ }^{5}$

L'Epistre très utile se compose de trois parties: une lettre à Marguerite de Navarre, une "Défense pour les femmes," et l'épître proprement dite qui dénonce les abus de l'Église papale ainsi que le manque de scrupule des pasteurs de Genève hostiles à Farel et Calvin. L'envoi l'annonce d'emblée, par-delà son désir de mobiliser la reine, l'ancienne abbesse prend la plume pour guider les femmes à "sçavoir et entendre la vérité": “. . . affin que désormais [les femmes] ne soyent en elles-mesmes ainsi tormentées et affligées, ains plustost resjouyes, consolées et esmeues à suyvir la vérité, qui est l'Evangile de Jésus-Christ" (a3 $3^{v}$; Herminjard, vol. 5, p. 298). ${ }^{6}$ La publication même de l'Epistre très utile constitue un acte délibéré de transgression des interdits de l'Église: les réformés ont beau encourager leurs épouses et leurs filles à lire l'Écriture, ils invoquent l'autorité de saint Paul pour leur refuser l'exercice de la prédication. Dans la première épître aux Corinthiens, l'apôtre observait:

Comme dans toutes les Églisses des saints, que les femmes se taisent dans les assemblées, car il ne leur est pas permis d'y parler; mais qu'elles soient soumises, selon ce que dit aussi la loi. Si elles veulent s'instruire sur quelque chose, qu'elles interrogent leurs maris à la maison, car il est malséant à une femme de parler dans l'Église (I Cor. 14, 34-36).

La première épître à Timothée (2,11-12) répète l'obligation des femmes de se taire dans l'église. Farel, Calvin et tous les prédicateurs de l'entourage immédiat de Dentière confirment l'interdit paulinien.

Dans sa lettre d'envoi, Dentière justifie donc son entreprise en invoquant le choix d'un public exclusivement féminin; les réformées sont en effet 
autorisées à commenter l'Écriture entre elles, en l'absence des hommes: "Et combien que [ne] nous soit permiz de prescher ès assemblées et églises publiques, ce néantmoins n'est pas deffendu d'escrire et admonester l'une l'aultre, en toute charité (a3; Herminjard, vol. 5, p. 297). À l'ouverture d'un livre tiré à 1500 exemplaires, la précision de Dentière à l'égard de l'auditoire visé fait ostensiblement figure de fiction rhétorique puisque la plupart des lecteurs de l'époque sont des hommes. Cette prétendue justification (qui ne pouvait tromper personne) se double d'un désir évident de narguer les pasteurs de Genève qui ont chassé Farel et Calvin: la mention explicite du sexe de l'auteur plutôt que de son nom dans le titre de l'oeuvre (Epistre très utile... faicte et composée par une femme) suggère ironiquement que dans ce temps de corruption, aucun prédicateur masculin n'est capable de porter la parole divine. L'humiliation du clergé genevois attaché à la cause de la Réforme ne saurait être plus complète: pour propager sa vérité, Dieu a eu recours à une femme, un être que toute l'Église considère comme inférieur et silencieux. Dentière adopte en somme le rôle de l'âne de Balaam châtiant son maître. L'épître proprement dite confirme cette interprétation; un passage parodie les réactions scandalisées que suscite invariablement la prédication des femmes: “. . . Dieu seroit bien iniuste nous auooir tant caché la verité, ha, il ne eust pas tant demouré la nous reueler et donner à cognoistre maintenant par lés femmes (dlv). Or il suffit, observe Dentière, de consulter les textes sacrés pour constater combien le mépris pour les femmes est contraire à l'esprit et à la lettre de l'Écriture (a4 $\left.4^{\mathrm{r}}\right)$.

L'ancienne abbesse regroupe les "preuves" de sa démonstration en réseaux cohérents, écartant d'avance toutes les objections possibles pour mettre en évidence la mauvaise foi (dans tous les sens de l'expression) du clergé. Les exemples de Sarah, Rébecca, Ruth, Déborah, et de tant d'autres héroïnes bibliques prouvent de manière éloquente le rôle des femmes, constate Dentière. De même, les écrits néo-testamentaires célèbrent ouvertement la Vierge, Élizabeth, la Samaritaine $\left(\mathrm{a} 4^{\mathrm{r}-\mathrm{v}}\right)$. Quant aux hommes, ils sont souvent plus à blâmer que les femmes. La misogynie est le résultat d'une lecture partielle et partiale des textes sacrés, elle se fonde sur des exclusions d'épisodes, des manipulations tendancieuses de la parole divine:

Je demande, fauldroit-il condamner Ruth, pourtant qu'elle est du sexe féminin, à cause que l'hystoire d'icelle est escripte en son livre? Je ne le pense pas, veu qu'elle est bien nombrée à la généalogie de Jésus Christ (Matt.1) ... S'il est question de parler des grâces qui ont esté faictes aux femmes, quelle plus grande a esté faicte à créature sus la terre que à la vierge 
Marie, mère de Jésus, d'avoir porté le filz de Dieu (Matt.1)?...Parquoy, que fault-il tant jaser des femmes? Veu que jamais femme n'a vendu ne trahy Jésus, mais un homme, nommé Judas. Qui sont ceux-là, je vous prie, qui ont tant inventé et controuvé de cérémonies, hérésies et faulses doctrines sus la terre, sinon les hommes? Et les paovres femmes par eulx ont esté séduictes. Jamais femme n'a esté trouvée faulx prophète, ouy bien par iceux trompée (a4 ${ }^{\mathrm{r}}-\mathrm{a} 5^{\mathrm{r}}$; Rilliet, pp. 379-380).

Ces arguments s'inscrivent, on le voit, dans le cadre de la Querelle des Femmes. ${ }^{7}$ Un des premiers jalons de ce débat, La Cité des dames (composé entre 1405 et 1407) de Christine de Pisan, s'appuyait déjà sur les mêmes passages bibliques que l'Epistre très utile pour plaider la cause des femmes et prouver l'égalité intellectuelle des deux sexes. ${ }^{8}$ Parmi d'autres textes qui relèvent d'une inspiration analogue, le De nobilitate et praecellentia foeminei sexus (composé en 1509 et publié en 1529) d'Henricus Cornelius Agrippa a, selon Irena Backus, exercé une influence directe sur le choix des références testamentaires de la "Défense pour les femmes" de l'Epistre très utile. ${ }^{9}$ Pour Agrippa et pour Dentière, s'impose en outre la volonté de poursuivre jusqu' au bout les conséquences sociales du sacerdoce universel proclamé par les partisans de la Réforme. Dans une culture fondée sur le respect du Verbe, comment en effet séparer l'homo religiosus de l'homo loquens? La parole reçue et transmise constitue le fondement même d'une telle conception religieuse; c'est elle qui établit le lien avec le divin et réunit la communauté. Ce n'est donc pas un hasard si l'envoi de l'Epistre très utile souligne l'efficacité de Marguerite de Navarre à diffuser l'idéal évangélique: l'oeuvre de Dentière ne fait à cet égard que réitérer le geste de la reine. Aux injonctions au silence se substitue l'appel à la prise de la parole par les femmes. Dans sa lettre à la reine, l'ancienne abbesse observe: "Car ce que Dieu vous a donné, et à nous femmes révélé, non plus que les hommes le debvons cacher et fouyr dedens la terre" (a3r; Herminjard, vol. 5, p. 297). La remarque était d'actualité: en cette même année de l'Epistre très utile (1539), l'imprimeur Gérard publie aussi un recueil de poèmes religieux de Marguerite de Navarre où figure le Miroir de l'ame pecheresse, qui avait échappé de justesse à la condamnation de la Sorbonne lors de sa première édition. "Nous femmes," rappelle dans ce sens Dentière à sa correspondante, "debvons sçavoir fuyr et éviter toutes erreurs, hérésies et faulses doctrines ... comme desjà assez par voz escriptz est démontré" (a2r; Herminjard, vol. 5, p. 296).

De manière semblable, dès les années 1520 , des femmes prennent part aux transformations religieuses du Saint Empire germanique. Auteur notamment 
d'une apologie du mariage des ministres du culte, Katherine Schütz-Zell semble même plus activement engagée que Dentière. À la fin de sa vie, elle résume en quelques phrases sa contribution à la communauté de Strasbourg dont son mari est pasteur: “... comment j'ai aidé à établir l'Évangile, recueilli l'exilé, réconforté les réfugiés sans abri, aidé l'Église, la prédication et les écoles, Dieu s'en souviendra, même si le monde oublie ou ne s'en aperçoit pas." ${ }^{10}$ En 1539, dans les milieux favorables au renouveau spirituel, les arguments et l'engagement religieux de Dentière sont donc loin de constituer un cas exceptionnel. Mais l'Epistre très utile se distingue de tentatives analogues par son agressivité. Dentière semble à dessein reproduire le ton des sermons de Farel dont le nom est mentionné dans l'épître proprement dite $\left(\mathrm{d} 6^{\mathrm{r}}\right)$. À l'instar du prédicateur, l'ancienne abbesse affirme au lieu de déduire, procède par contrastes violents, par simplifications outrancières. Invectives indignées et blasphèmes se mêlent à des citations testamentaires pour arracher les faux semblants, dénoncer l'imposture de l'Église romaine. Ces caractéristiques apparaissent bien dans un passage de l'épître proprement dite qui s'attaque aux abus des papistes: "Exercez vostre office, tyrans! rompezvous la teste contre la pierre! Rien n'y ferez, bedeaux du Pape, sinon la vous rompre. Vous serez froisséz. Car en tuant et bruslant le corps, n'avez nulle puissance sus l'âme" (b6'; Herminjard, vol. 5, p. 299). L'impétuosité des accusations et leur qualité orale rappellent directement les assauts de Farel tels que cette diatribe contre les détracteurs des réformés: "Prenez yeuz, Chrestiens, et ne vous arrestez plus à ces menteurs! Délaissez-les comme lépreux et vrayement frappez de la ladrerie de Giezy, de laquelle Viret et ses semblables par la grâce de dieu sont purs."11

Nul n'a tonné avec plus de véhémence que Farel, rapportent les témoignages d'époque. ${ }^{12}$ Dans une lettre datée du 19 août 1524 , Oecolampade reproche au prédicateur d'abreuver les prêtres de torrents d'injures: "Tu as été envoyé pour évangéliser, non pour maudire" (Evangelizatum, non maledictum missus es). ${ }^{13}$ Ou encore dans le Summaire et briefve declaration (1525), dont Dentière ne pouvait ignorer l'existence puisque cet ouvrage constitue le premier texte français à définir les points essentiels de la doctrine réformée (les rééditions de $1534,1542,1552$ attestent son succès en contrée romande), Farel s'en prend au clergé de l'Église romaine: "Faictes-vous ce deshonneur à Dieu que son oeuvre soit cachee à ses enfantz comme nuysible et mortelle?"14 Animée par le même esprit belliqueux, Dentière injurie les théologiens: "Siavezl'Escripture pour vous, aveugles et conducteurs d'aveugles, que ne le monstrez-vous?" (blv; Herminjard, vol. 5, p. 298): 
Ilz sont tant de docteurs, tant de sages, tant de grans clers, tant d'universitez contre nous, paovres femmes, qu[i] sommes rejectées et mesprisées de tout le monde. A quoy servent-ilz, je vous prie? S'ilz ne veullent monstrer leur cause estre bonne et ordonnée de Dieu, endurez-vous qu'ilzdominent sur vous? Nous disons le contraire d'eux, qu'ilz le preuvent. Nous le voulons monstrer, qu'ilz se deffendent par l'escripture saincte ... il semble qu'ilz ne soyent ordonnez que pour triumpher, dancer, braguer, ivrongner, et paillarder. . . (b2'; Rilliet, p. 381).

"Faictes que la trompette du sainct Evangile soit oye jusques à la fin et bout du monde," proclamait pour sa part Farel dans le Summaire et briefve declaration: "Donnez vertu aux vrays evangelisateurs. Destrui[s]ez tous semeurs d'erreur, affin qu[e] toute la terre vous serve, vous invoque, vous adore et vous honore" (p. 248). Le modèle du miles christianus de Farel, si pleinement convaincu de sa mission qu'il attaque sans pitié tout ce qui lui fait résistance, semble bien avoir inspiré le ton agressif de l'Epistre très utile. À l'instar du prédicateur, Dentière s'en prend avec fougue aux contraintes arbitraires. La description du bon pasteur du Summaire s'applique parfaitement à l'attitude de Dentière, à la différence près que ce bon pasteur n'est jamais, pour Farel, de sexe féminin et ne prêche jamais la cause des femmes: “. . . ne souffrant qu' on mette en nouvelle subjec[t]ion par tradition ou o[r]donnance humaine les brebiz affranchies par nostre Seigneur Jesus" (p. 232).

Dans une optique analogue, si, comme le suggère Irena Backus, l'aspect superficiel des connaissances théologiques de Dentière s'explique par le type d'enseignement prodigué à des femmes même abbesses, ${ }^{15}$ il n'est pas exclu de voir dans ces lacunes une conséquence directe de la conception religieuse de Dentière. Pour Farel (qui n'était ni théologien, ni prêtre de formation) comme pour Dentière, loin d'être une question d'érudition livresque, l'enseignement religieux postule inévitablement une action directe sur la société pour la conformer à l'Évangile. L'oeuvre de "vulgarisation" de Farel, ses adaptations en français de manuels d'éducation religieuse pour les simples illustrent bien cet effort de diffusion concrète de la parole divine.

Dans l'Epistre très utile de Dentière, l'image traditionnelle de la chrétienne modeste, passive et silencieuse cède donc la place à l'idéal de la femme étroitement associée aux efforts des prédicateurs pour inscrire la foi dans la réalité sociale. On pourrait qualifier d'organique la congrégation telle que la conçoit Dentière. Elle suppose l'absence de discrimination de tout ordre, même sexuelle, entre les membres d'un groupe animé par une spiritualité commune. Dans l'épître proprement dite, Dentière interroge ses lecteurs: 
Avons-nous deux Evangiles, l'un pour les hommes, et l'aultre pour les femmes? L'un pour les sages, et l'aultre pour les folz? Ne sommes-nous pas un en nostre Seigneur? Au nom duquel sommes-nous baptisez, de Pol ou d'Apollo, du Pape ou de Luther (dl'; Rilliet, p. 383)?

C'est en observant scrupuleusement l'Écriture que l' ancienne abbesse propose ce modèle religieux à contexture égalitaire. Le premier épître aux Corinthiens $(1,10)$ s'en prenait déjà à ceux qui se réclamaient de Paul ou d'Apollos, et non du Christ. Le même appel à l'union se retrouve dans l'épître aux Galates: "Car vous êtes tous fils de Dieu par la foi en Jésus-Christ; vous tous qui avez été baptisés en Christ, vous avez revêtu Christ. Il n'y a plus ni Juif, ni Grec, il n'y a plus ni homme ni femme; car vous êtes un en Jésus-Christ (Gal. 3, 26-28).

Dentière est loin d'être la seule à évoquer ces passages néo-testamentaires pour défendre les femmes. Parmi d'autres exemples proches de l'ancienne abbesse, Agrippa y avait déjà eu recours dans le même dessein. ${ }^{16}$ Mais au lieu de considérer l'égalité des chrétiens sur un plan purement théorique, Dentière l'envisage au contraire comme un but à réaliser hic et nunc. Un ouvrage attribué à Jeanne de Jussie permet de suivre les réactions des adversaires de la Réforme à l'arrivée de Dentière. Intitulé a posteriori Le levain du calvinisme, le récit de cette Clarisse de Génève relate l'intrusion de Farel et de ses auxiliaires dans le couvent de Sainte-Claire, le 25 août 1535 . Un passage évoque en ces termes le rôle de Dentière:

En celle compagnie estoit une Moine Abbesse fausse, ridee, \& langue diabolique, ayant mari \& enfans, nommee Marie d'Entiere, de Picardie, qui se mesloit de prescher, \& de peruertir les gens de deuotion. . . pour l'enuie que elle auoit d'en peruertir aucune [religieuse], ne faisoit conte des reproches, \& disoit, hé pauures creatures! si vous sçauiez qu'il fait bon estre aupres d'vn ioly mary, \& comment Dieu l'a aggreable, I'ay long temps esté en ces tenebres $\&$ hypocrisie où vous estes, mais le seul Dieu m'a faict cognoistre l'abusion de ma chetiue vie, $\&$ suis paruenue à la vraye lumiere de verité. ${ }^{17}$

Ces propos si choquants aux yeux de la narratrice se retrouvent dans la plupart des textes de contemporains opposés à l'Église romaine. La critique de la vie contemplative et récluse de la nonne qui (la suite du Levain l'indique) ignore l'Écriture, le motif des vocations forcées qui fait l'objet de controverses ultérieures entre les Clarisses et les envahisseurs apparaissent déjà chez les évangéliques et notamment dans les colloques matrimoniaux d'Érasme. ${ }^{18}$ Plus que du sens du discours prêté à Dentière, le scandale provient, dans ce passage, 
du ton sur lequel ces mots sont prononcés. Il est liéa l' adoption d'une "méthode de choc" du prosélytisme, à la détermination de Dentière d'entraîner l'auditoire de gré ou de force vers la vérité testamentaire. Le récit renforce à dessein le contraste entre l'énergique agressivité de Dentière et la passivité silencieuse des Clarisses; il met en évidence le refus de l'abbesse de prêter la moindre attention aux reproches des nonnes offusquées. La nouveauté de l'intervention de Dentière dans le couvent ce jour-là est en définitive dans l'appropriation par une femme du modèle de la prédication donné par Farel.

\section{Les effets de la prédication des femmes}

Quelles sont les conséquences de l'action de Dentière? Tous les exégètes s'accordent sur un point: les tentatives de cet ordre ne modifient en rien le statut des femmes; leur subordination à l' autorité masculine et à la loi du silence dans l'Église persistent durant des siècles..$^{19} \mathrm{Au}$ couvent de Sainte-Claire, la fougue de Dentière ne convaincra qu'une seule religieuse qui était d'ores et déjà acquise à la cause de la Réforme puisqu'elle lisait la Bible à l'insu de ses compagnes. Les femmes ne sont alors prêtes ni à leur libération, ni à une prise de conscience collective.

Si les nonnes genevoises refusent farouchement le changement, quelle est alors la réaction de Farel et de Calvin dont Dentière défend si ardemment la cause? Conformément à la pensée réformée, le Summaire et briefve declaration de Farel n'envisage jamais la possibilité de la prédication féminine: $c$ 'est au foyer, dans son rôle de mère et d'épouse que la femme est le meilleur auxiliaire de l'homme (p. 275). Mais comment oublier qu'en dépit de cette conception Farel se fait accompagner de Dentière lors de l'intrusion dans le couvent de Sainte-Claire et lui laisse prendre la parole en sa présence? Le silence de Farel durant le discours de Dentière ne peut s' interpréter que comme le signe d'un accord implicite. De manière analogue, l'attitude de Calvin (dont l'Epistre très utile réclame aussi le retour à Genève) à l'égard de la prédication des femmes est peut-être moins négative qu'il n'y paraît au premier abord. Le théologien est, on le sait, loin d'être favorable à la participation active des femmes à la vie religieuse. ${ }^{20} \mathrm{Parmi}$ d' autres manifestations frappantes de cette réticence, le sermon de Calvin sur le chapitre 3 de l'épître aux Galates interprète les versets 26-28 (..."il n'y a plus ni homme ni femme; car vous êtes un en Jésus-Christ. ...") sous la forme d'une prédiction qui se réalisera après la vie humaine. Sans nier l'égalité essentielle des sexes dans le domaine spirituel, Calvin n'admet pas, comme le fait Dentière, sa réalisation sociale: 
Or cependant sainct Paul n'a pas voulu dire, quant à la police de ce monde, qu'il n'y ait des degrés divers: car nous sçavons qu'il y a des serviteurs et maistres: il y a des magistrats et peuples suiets: il y a au mesnage l'homme qui est le chef, et la femme qui luy doit estre suiete: nous sçavons donc que cet ordre-là est inviolable et nostre Seigneur Iesus Christ n'est pas venu au monde pour faire une telle confusion que ce qui est establi de Dieu son Pere soit aboli. . . Et cependant si nous venons à la vie celeste, que nous cognoissions que là tout ce qui est du monde s'escoule et s'esvanouit. . . ${ }^{21}$

Dans la même optique, le commentaire du chapitre 11 de la première épître aux Corinthiens (versets 4-10) attribue une valeur illustrative à une allusion de saint Paul à la prédication des femmes. Sans songer un instant à remettre en cause les textes où l'apôtre interdit formellement la prophétisation des femmes (I Cor. 14, 34-36; I Tim. 2, 11-12), Calvin tente au contraire de conformer cette apparente exception à la règle énoncée ailleurs en lui donnant la fonction d'un simple exemple:

Il [saint Paul] dit que si une femme prie ou prophetize la teste descouverte, elle deshonore son chef.... il pourroit sembler que sainct Paul repugne à ce qu'il dict en l'autre passage: à sçavoir qu'il n' est poinct permis à une femme d'enseigner. Si cela est vray, comment luy attribue-t-il ici la charge et office de prophetizer. La response à cela est que sainct Paul n'a point voulu mettre ici les femmes en chaire, mais il a posé le cas, comme nous avons accoustumé de faire, etc'est un langage assez commun. Posons le cas qu' une femmes preschast; ou qu'elle fist les prieres au nom de tous ... et qu'en un tel acte elle fust deschevelee, que seroit-ce? chacun auroit un tel spectacle en horreur. ${ }^{22}$

Malgré ce manque de sympathie évident pour la prédication des femmes, on aurait pourtant tort de conclure sur un constat négatif trop radical. Nulle part Calvin ne condamne les femmes qui prêchaient à Genève avant son retour dans la ville en 1541 . Or il ne pouvait ignorer leur pratique. Outre l'intervention de Dentière, Le levain du calvinisme de Jussie évoque en effet la "langue diabolique" et les sermons de la genevoise Claudine Levet lors d'une intrusion des réformés dans le couvent de Sainte-Claire. En guise de confirmation de ce récit, les Actes et gestes merveilleux de la cité de Genève (1554) de Froment rapportent qu'au cours des années 1530 la même dame Levet prêche devant des assemblées d'hommes et de femmes. Froment ne cache pas son admiration pour les dons de cette prédicante qui semble avoir joui d'une véritable notoriété. 23

Jane Dempsey Douglass suggérait déjà que l'activité de Levet et Dentière 
aurait pu déterminer un commentaire de Calvin sur le sermon de la première épître aux Corinthiens $(14,34)$ où le théologien admet la prédication des femmes à des moments exceptionnels. ${ }^{24} \grave{A}$ cette remarque, je voudrais ajouter une autre observation, directement liée au prosélytisme militant de Dentière (et de Levet), et qui semble confirmer l'hypothèse de Douglass. Elle consiste à interroger les textes de Calvin contemporains de ces prêches et de la parution de l'Epistre très utile: de quelle manière Calvin aborde-t-il à cette époque la question du silence des femmes? En 1541, de retour à Genève à la fin de son exil, Calvin publie chez Jean Gérard (qui, deux ans auparavant avait imprimé l'Epistre très utile) sa propre traduction de l'édition princeps de l'Institution de la religion chrestienne (1536) et la dédie à François $1^{\text {er }}$. De caractère plus accessible que l'érudite oeuvre latine, la version française s'adresse à un large public, ${ }^{25}$ mais suit cependant de très près l'ouvrage initial. Cette fidélité se vérifie notamment dans les commentaires sur la prédication des femmes qui rendent mot pour mot le texte latin. ${ }^{26}$

Dans ce passage, Calvin distingue deux types de règles. La première catégorie a trait à la "police" sociale. Le sens de ces édits de nature séculière, précise Calvin, est relatif aux exigences du groupe qui les formule: il varie selon les configurations sociales, mais chaque membre de la communauté est néanmoins tenu de s'y conformer pour maintenir l'ordre et la paix générale. Parmi les exemples de ce premier type de lois figurent le silence des femmes et d'autres coutumes tels que l'agenouillement des arthritiques ou la nécessité pour les femmes de se couvrir la tête. Ces lois ne sont en rien liées à celles de la seconde catégorie qui concernent le dogme religieux. Enfreindre les interdits du premier groupe n'est donc pas, remarque Calvin, un bien grand délit, et ne met en danger ni la piété ni le salut du chrétien. Dans certains cas, l'infraction aux lois du premier type peut même constituer une nécessité:

Nous avons les exemples de la première espèce [le respect de lois relevant de convenances et d'ordre social] en S. Paul, quand il deffend aux femmes d'enseigner publiquement et de ne se montrer sinon la teste couverte. Et en avons de quotidiens entre nous, comme de prier publiquement à genouilz, et à nue teste... Quoy? y a-t-il quelque si grand mistère en la coiffure d'une femme, que ce soit un grand crime de sortir en la rue nue teste? Le silence luy est-il tellement commandé, qu'ellene puisse parler sans grande offence? ... Non certes. Car si la necessité de son prochain la pressoit, tellement qu'elle n'eust loysir de se coiffer, elle ne pèche en rien, si elle accourt nue teste pour luy ayder, et l'heure arrive quelque fois, qu'il luy vauldroit mieux parler, que se taire [je souligne]. ${ }^{27}$ 
L'activité de Marie Dentière a-t-elle été étrangère à ces réflexions? La question mérite d'être posée. On ne peut en effet s'empêcher de lire dans ces précisions une justification d'autant plus nette du rôle de l'ancienne abbesse qu'elle paraît dans un ouvrage en français et est par conséquent à la portée de nombreux réformés de Genève. Dans ce sens, l'Epistre très utile aurait bien pu contribuer à attirer l'attention du réformateur sur la possibilité d'associer les femmes à sa mission d'édification, de leur permettre, à certaines occasions, d'instruire leur prochain pour construire une communauté chrétienne. Comme l'indique l'absence de précision de l'expression "l'heure arrive quelque fois," la concession de Calvin se situe sans aucun doute sur un plan théorique et abstrait. Elle constitue néanmoins un premier pas, timide mais difficilement récusable, vers la remise en cause du silence des femmes dans l'Église.

\section{University of California, Santa Barbara}

\section{Notes}

1. Je remercie Mary McKinley de m'avoir généreusement communiqué une copie de l'Epistre très utile et d'avoir suivi avec tant d'attention l'élaboration de cet essai. Pour une vue générale sur la question de la prédication des femmes, voir parmi d'autres ouvrages, Nathalie Zemon Davis, Society and Culture in Early Modern France (Stanford, Stanford University Press, 1975), pp. 93 et sq; Évelyne Berriot-Salvadore, Les femmes dans la société française de la Renaissance (Genève, Droz, 1990); et Ruth Kelso, Doctrine for the Lady of the Renaissance (Urbana, University of Illinois Press, 1956). Sur Marie Dentière, voir Aimé-Louis Herminjard, Correspondance des réformateurs dans les pays de langue française, vol. 6, 1539-1540 (Genève, Georg, 1883), pp. 173-174, n. 28; Thomas Head, "A Propagandist for the Reform: Marie Dentière," dans K. Wilson, éd. Women Writers of the Renaissance and Reformation (Athens, University of Georgia Press, 1987), 260-283; Madeleine Lazard, "Deux soeurs ennemies, Marie Dentière et Jeanne de Jussie: nonnes et réformées à Genève," dans Les réformes. Enracinement socio-culturel, études réunies par B. Chevalier et C. Sauzet (Paris, La Maisnie, 1985), 239-249; Irena Backus, "Marie Dentière: un cas de féminisme théologique à l'époque de la Réforme?," Bulletin de la Société de l'Histoire du Protestantisme Français (1991), 177-195.

2. La guerre et deslivrance, éd. par A. Rilliet, "Mémoires et documents publiés par la Société d'Histoire et d'Archéologie de Genève," vol. 20 (Genève, Jullien, 1879-1888), pp. 309-384.

3. Herminjard, vol. 5, pp. 295-296, n. 2 .

4. Gabrielle Berthoud, Antoine Marcourt, réformateur et pamphlétaire (Genève, Droz, 1973), pp. 65-70.

5. Herminjard, vol. 5, p. 296, n. 3.

6. Toutes les références à l'Epistre très utile renvoient à l'édition conservée au Musée Historique de la Réformation de l'Université de Genève, sous la cote D.Den.1A. Afin de 
faciliter la lecture, j'ai résolu les abréviations. Des extraits de l'Epistre ont été publiés par Herminjard, vol. 5, pp. 295-304 et Rilliet, pp. 378-384. Comme l'édition originale de l'Epistre très utile est rarissime, j'ajoute aussi, lorsqu'elles existent, les références aux extraits de Herminjard et de Rilliet. Les passages retenus par ces deux exégètes constituent jusqu'ici les seules rééditions de l'Epistre. Pour le choix de leurs extraits, voir Mary McKinley, "The Absent Ellipsis. The Edition and Suppression of Marie Dentière in the XVIth and XIXth Centuries," dans Women Writers in Pre-Revolutionary France: Strategies of Emancipation, sous la dir. de Colette Winn (New York, Garland, 1997).

7. Sur la Querelle des Femmes, voir le Débat sur le Roman de la Rose, sous la dir. de Eric Hicks (Paris, Champion, 1977).

8. Christine de Pisan, Le livre de la Cité des Dames, édition modernisée par T. Moreau et E. Hicks (Paris, Stock, 1986), Deuxième Livre, chapitres 31, 32, 38 à 40, et Troisième Livre, chapitres 2 à 17.

9. Backus, pp. 184-187.

10. Katherine Zell, Ein Brief and die genze Burgerschaft der Stadt Strassburg betreffend Hern Ludwig Rabus (1557), reproduit dans J. C. Füssli, Beytrage zu Erlaüterung der KirchenReformationsgeschichte des Schweitzerlandes, vol. 5 (Zurich, Heidegger und Comp., 1753), pp. 191-192. C'est moi qui traduis. Voir aussi Paul Russell, Lay Theology in the Reformation: Popular Pamphleteers in Southwest Germany (Cambridge, Cambridge University Press, 1986), pp. 189-211.

11. Herminjard, vol. 2, lettre 402, p. 481. Dans ce passage, Farel s'en prend aux accusations portées contre Viret.

12. Guillaume Farel, 1489-1565 (Paris/Neuchâtel, Delachaux et Niestlé, 1930), p. 22.

13. Herminjard, vol. 1, lettre 115, p. 265.

14. Guillaume Farel, Summaire et briefve déclaration, éd. A.-L. Hofer (Neuchâtel, BelleRivière, 1980), p. 246. Toutes les références renvoient à cette édition.

15. Sur la culture théologique de Dentière, voir Backus, pp. 183-195.

16. Henricus Cornelius Agrippa, De nobilitate et praecellentia foeminei sexus, éd. dirigée par A. Antonioli (Genève, Droz, 1990), pp. 118-119.

17. Jeanne de Jussie, Le levain du calvinisme ou commencement de l'hérésie de Genève, éd. A.C. Grivel (Genève, Fick, 1865), pp. 173-174.

18. Lazard, pp. 239-249.

19. Davis, pp. 93-95.

20. John Lee Thompson fait observer dans John Calvin and the Daughters of Sarah: Women in Regular and Exceptional Roles in the Exegesis of Calvin, his Predecessors, and his Contemporaries (Genève, Droz, 1992), pp. 40-45, que Calvin ne devait pas avoir Froment et Dentière en grande estime à cause notamment des activités lucratives de Froment et de sa vénalité, et que, par conséquent, Calvin n'avait probablement pas lu l'Epistre très utile. Cette conclusion me paraît assez hâtive; quatre siècles plus tard, il est évidemment 
impossible de connaître l'opinion de Calvin sur Dentière en tant qu'individu, mais il est difficile de croire que le réformateur ne suivait pas avec attention les événements religieux d'une ville dont il était exilé, surtout lorsque ces événements concernaient directement ses convictions et impliquaient les membres de son entourage. Voir aussi Thompson, pp. 269280, sur la position de Calvin à l'égard des femmes.

21. Jean Calvin, Opera quae sunt supersunt omnia, éd. G. Baum, E. Cunitz, E. Reuss, "Corpus Reformatorum” (Brunswick et Berlin, Schwetschke \& filium, 1863-1900), vol. 50, pp. 567568.

22. Calvin, vol. 49 , p. 725.

23. Jussie, p. 185; Antoine Froment, Les actes et gestes merveilleux de la cité de Genève, éd. G. Revilliot (Genève, Fick, 1854), pp. 15-21.

24. Jane Dempsey Douglass, Women Freedom and Calvin (Philadelphia, Westminster Press, 1985), pp. 30-64, 105-107; cette interprétation est reprise par Thompson, p. 205.

25. Le caractère accessible du texte français de 1541 se remarque notamment dans l'élimination des termes savants de l'édition de 1536. Jean Calvin, Institution de la religion chrestienne, éd. Jacques Pannier (Paris, Les Belles Lettres, 1961), vol. 1, pp. xviii-xxiv.

26. Pour la version latine de ce passage dans l'édition de 1536, voir Jean Calvin, Opera selecta, éd. P. Barth et W. Niesel (Munich, Kaiser, 1926-1962), vol. 1, pp. 255-258. Voir aussi Douglass, p. 30.

27. Jean Calvin, Institution de la religion chrestienne, vol. 4, pp. 193-194. 